\title{
Storming the Tower of Babel
}

\author{
The search for life among the stars stepped up a gear last week with the \\ First Astrobiology Science Conference in California. Henry Bortman and \\ Philip Ball were there to test the atmosphere.
}

f scientific disciplines are usually conceived naturally, as like-minded researchers converge on an emerging idea, then astrobiology is more like a test-tube baby. This new field arose out of NASA administrator Dan Goldin's desire to make the search for extraterrestrial life one of the central themes for his agency.

Last week, some 500 scientists gathered at NASA's Ames Research Center in Moffett Field, California, to help map out an agenda for the newly emerging field. Most left cautiously optimistic about the discipline's future, but facing a difficult challenge: how to unite the diverse strands of research collected under astrobiology's umbrella. And, in particular, how to get the various specialists, each with their own research agendas and jargon, talking to one another in a meaningful way.

\section{Breaking the mould}

Some of the scientists at last week's meeting had gathered together before. But at earlier conferences, such as the six Bioastronomy meetings, they attended separate sessions where, for example, biologists would speak to biologists. The First Astrobiology Science Conference tried to break this mould with cross-disciplinary sessions along themes such as "Water: the sine qua non of life".

This made for a diverse set of presentations. Jody Deming, from the University of Washington in Seattle, discussed a new technique for studying the microorganisms that live inside tiny hypersaline pockets of brine within Arctic sea ice. These 'extremophiles' can survive at temperatures as low as $-15^{\circ} \mathrm{C}$, and are interesting because they live in conditions that might resemble those on Europa, one of Jupiter's icy moons.

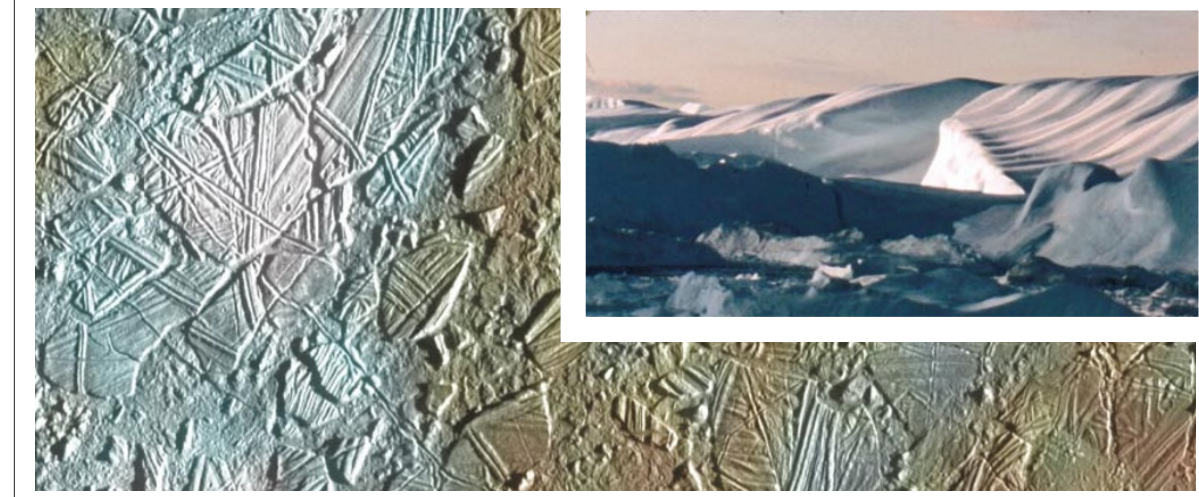

Life in the freezer: conditions in Arctic ice (inset) may mirror those on the surface of Europa.

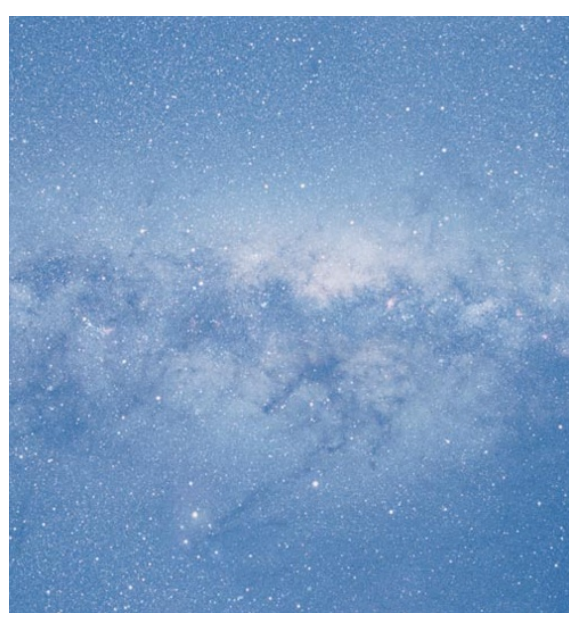

Barren: is our galaxy's core devoid of life?

Bob Haberle of NASA Ames, meanwhile, presented a climate model for Mars. $\mathrm{He}$ showed that local pressure and temperature conditions on 29 per cent of the planet's surface could, in theory, enable liquid water to exist on the surface for minutes or maybe even hours at a time.

Other researchers considered the prospects for extraterrestrial life on a grander scale. While the 'habitable zone' of our Solar System is a well-established concept, Peter Ward, Donald Brownlee and Guillermo Gonzalez from the University of Washington have assessed the chances of such zones existing on a galactic scale. They argued that the outer reaches of a galaxy similar to our Milky Way would not contain sufficient quantities of heavy elements, such as silicon, aluminium and iron, to form the rocky, tectonically active planets thought to be a prerequisite for life. Near the centre of such galaxies, the dense packing of stars means that planets are likely to be heavily bombarded by comets and bathed in hazardous radiation from supernovae. Most galaxies, the team concluded, will contain only limited regions where life is possible, and many may be utterly barren.

\section{Teething troubles}

Opinions differ on how successful the meeting was at integrating such diverse approaches into a coherent whole. "Differences in approaches between disciplines can provide fresh insights," says Jack Lissauer, a planetary scientist at NASA Ames. "If you look at a planet from a purely planetary-science perspective, you can look at 99.9 per cent of the mass, but can be missing the atmosphere completely. Yet that is crucial to habitability," he says.

On occasions, however, it was clear that communications had broken down. For instance, after one detailed presentation on evolutionary relationships among the Archaea, thought to represent some of the most primitive organisms on Earth, one bemused astronomer rose from his seat to ask: "Why should I be interested in this?"

Given the circumstances of the field's genesis, some delegates at last week's meeting believe such glitches are inevitable. "Astrobiology is kind of an artificial conglomeration," says Jay Melosh, a theoretical geophysicist at the University of Arizona in Tucson. "Unlike biochemistry, which grew organically, this field was invented by Dan Goldin saying: "This is going to be the field of astrobiology'."

Coordinating the field will be the task of NASA's Astrobiology Institute, an 'institute without walls' based at Ames but embracing a diverse set of research groups across the United States. While its task may be formidable, last week's meeting showed that astrobiology already has a respectable number of converts. Lynn Rothschild, a biologist at Ames who organized the meeting, says that attendance was twice as high as she originally anticipated.

"This may be remembered as the great ideas conference," concludes Melosh. "Out of that diversity there will be some ideas that get put together and perhaps grow and bear fruit. I'm not sure I can predict what's going to happen and what's not, but it will be interesting to watch." Henry Bortman is a freelance writer in San Francisco; Philip Ball is a Consultant Editor of Nature. - http://astrobiology.arc.nasa.gov/conferences/2000/ ABSciConf/index.html

NATURE|VOL 404 13 APRIL 2000| www.nature.com 